ORIGINAL ARTICLE

\title{
Defining the molecular basis of oncogenic cooperation between TAL1 expression and Pten deletion in T-ALL using a novel pro-T-cell model system
}

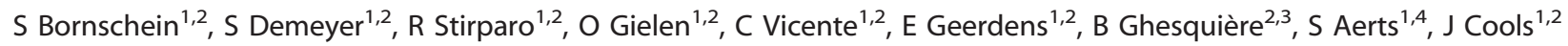 \\ and CE de Bock ${ }^{1,2}$
}

T-cell acute lymphoblastic leukemia (T-ALL) is caused by the accumulation of multiple mutations combined with the ectopic expression of transcription factors in developing $\mathrm{T}$ cells. However, the molecular basis underlying cooperation between transcription factor expression and additional oncogenic mutations in driving T-ALL has been difficult to assess due to limited robust T-cell model systems. Here we utilize a new ex vivo pro-T-cell model to study oncogenic cooperation. Using a systems biological approach we first dissect the pro-T-cell signaling network driven by interleukin-7, stem cell factor and Notch1 and identify key downstream Akt, Stat, E2f and Myc genetic signaling networks. Next, this pro-T-cell system was used to demonstrate that ectopic expression of the TAL1 transcription factor and Pten deletion are bona-fide cooperating events resulting in an increased stem cell signature, upregulation of a specific E2f signaling network and metabolic reprogramming with higher influx of glucose carbons into the tricarboxylic acid cycle. This ex vivo pro-T-cell system thereby provides a powerful new model system to investigate how normal T-cell signaling networks are perturbed and/or hijacked by different oncogenic events found in T-ALL.

Leukemia (2018) 32, 941-951; doi:10.1038/leu.2017.328

\section{INTRODUCTION}

T-cell acute lymphoblastic leukemia (T-ALL) is an aggressive hematologic malignancy, characterized by high white blood cell counts and infiltration of immature $T$ cells into the bone marrow and other tissues. T-ALL patients frequently display mutations in genes involved in signaling pathways that regulate T-cell development, including the NOTCH1 pathway, the IL7R-JAK-STAT signaling pathway (IL7R, JAK1/3 and STAT5) and the T-cell receptor-signaling pathway (AKT, PTEN and RAS). ${ }^{1,2}$ Moreover, T-ALL patients show mutually exclusive ectopic overexpression of HOXA, NKX2-1, TLX1/3 or TAL1 transcription factors. ${ }^{3,4}$ However, many of the in vitro cell model systems available to study how the expression of transcription factors and co-occurring mutations lead to the transformation of normal $T$ cells to cytokineindependent growth have a number of limitations.

Currently, the functional consequence of oncogenic lesions found in T-ALL is often carried out using in vitro cytokinedependent cell lines. For example, the ability of mutations to transform the interleukin (IL)3-dependent murine Ba/F3 cell line to cytokine-independent growth. However, the majority of these in vitro systems are either not physiological (that is, the pro-B Ba/ F3 cell line), rapidly lose cytokine dependency (that is, the MOHITO cell line $)^{5}$ or require the $T$ cells to be grown in the presence of a feeder-cell-dependent culture system (for example, OP9-DL1) in which additional signals delivered by OP9 are difficult to assess. ${ }^{6}$ Furthermore, the use of human T-ALL cell lines is limited due to the numerous genomic lesions already present making them difficult to assess early transformation events.
Normal T-cell development requires the complex interplay between developing progenitor cells and the thymic microenvironment. ${ }^{7}$ Early T-cell progenitors mature from CD4/CD8 double-negative (DN) cell into CD4/CD8 double-positive (DP) cells and then to CD4 or CD8 single-positive cells via exposure to soluble cytokines, including IL2 and II7, stem cell factor (Scf) and hedgehog ligands. Controlled Notch signaling is also critical for T-cell development, with deletion of Notch1 in murine hematopoietic stem and progenitor cells leading to a block in T-cell differentiation. ${ }^{8,9}$

Recently, a feeder-cell-independent system for the long-term culture of primary T-cell precursors has been described. ${ }^{10,11}$ Using a systems biological approach, we have used this pro-T-cell culture system to dissect the transcriptional networks induced by external cytokine stimuli. This pro-T-cell system was then used to dissect the molecular basis underlying the cooperation between ectopic overexpression of TAL1 and Pten deletion, frequently found in T-ALL patients.

\section{MATERIALS AND METHODS}

Pro-T-cell culture

Pro-T-cell cultures were established as described previously ${ }^{10}$ from C57BL/6 (Charles River Laboratories, Saint-Germain-Nuelles, France) or Rosa26-Cas9 knock-in transgenic mice (024858, Jackson Laboratories, Bar Harbor, ME, USA).

\section{Phospho-flow cytometry}

Phosphorylated proteins were stained using anti-Akt pS473-PE (Miltenyi Biotech, Cambridge, MA, USA), anti-STAT3 pY705-PE, anti-mTOR pS2448-PE and anti-Stat5 pY694-APC (eBioscience, San Diego, CA, USA). For Mct4 
staining of pro-T cells, cells were fixed using IC fixation buffer (eBioscience), followed by staining with anti-Mct4 antibody conjugated to Alexa-647 fluorochrome (clone D-1; Santa Cruz Biotechnology, Dallas, TX, USA). Cells were analyzed on a FACSCanto flow cytometer or FACS Verse (BD Biosciences, Bedford, MA, USA). Data were analyzed with FlowJo software (Tree Star, Ashland, OR, USA).

\section{RNA-seq expression analysis}

RNA extraction was carried out as described previously. ${ }^{12}$ The single-end RNA-sequencing data were first cleaned with the fastq-mcf software (https://github.com/ExpressionAnalysis/ea-utils) and quality control was performed with FastQC. Reads were mapped to the Mus Musculus (mm10) genome with Tophat2. To identify the gene expression HTSeq-count was used to count the number of reads per gene. These read count numbers were then normalized to the sample size. Differential gene expression analysis was performed with the R-package DESeq2 (https://git.bioconduc tor.org/packages/DESeq2). RNA-sequencing data were deposited within Gene Expression Omnibus (GSE98899).

Independent genetic clustering of RNA-sequencing results K-means clustering was performed using Multiple Experiment Viewer (http://mev.tm4.org). For the prediction of cis-regulatory features and cisregulatory modules 'i-cisTarget' was used and for gene ontology enrichment analysis, Gorilla.

Metabolic assays and liquid chromatography-mass spectrometry Pro-T cells were cultured for $24 \mathrm{~h}$ in $10 \%$ glucose-free RPMI medium supplemented with ${ }^{13} \mathrm{C}$-labeled glucose $(2 \mathrm{~g} / \mathrm{l})$. Metabolite measurements were performed using a Dionex UltiMate 3000 LC System (Thermo Scientific, Carlsbad, CA, USA) in-line connected to a Q-Exactive Orbitrap mass spectrometer (Thermo Scientific).

A detailed description of all methods used in this study is given in the Supplementary Materials and Methods.

\section{RESULTS}

Ex vivo pro-T cells recapitulate DN thymic T cells

A recently developed ex vivo culture system has been described that allows for feeder-cell-independent differentiation of hematopoietic stem and progenitor cells into pro-T cells. ${ }^{10,11}$ Here hematopoietic stem and progenitor cells are cultured in the presence of Scf, II7 and immobilized plate-bound DII4 (Figure 1a). Weekly analysis of the cell population showed T-cell differentiation comparable to thymic development in vivo. After 3 weeks, three different precursor populations (DN1, DN2 and DN3), defined by CD44 and CD25 expression, were detectable (Figure 1b). After 5 weeks, the major population (90-95\%) consisted of DN2 pro-T cells (lineage-negative, c-kit ${ }^{+}, \mathrm{CD} 44^{+}$, $\mathrm{CD}_{25^{+}}$) with cytoplasmic, but not surface, expression of CD3 (Figure $1 \mathrm{~b}$; Supplementary Figure 1). The cells remained negative for cell surface CD4 and CD8 expression (Supplementary Figure 1). The robust expansion and survival of pro-T cells depended on continuous DII4, Scf and II7 signaling with removal of any one of the three factors sufficient to abolish cell expansion and resulted in eventual cell death after 8 days (Figure 1c).

Notch, Scf and II7 signaling pathways regulate mutually exclusive gene clusters in driving pro-T-cell proliferation

We hypothesized that the individual Notch, Scf and II7 signaling pathways would induce a different set of genes to drive and regulate T-cell development and proliferation. To this end, pro-T cells were stimulated with each factor individually or with one of them removed from the culture before RNA-seq analysis (Supplementary Figure 2). These conditions did not result in any differences in the expression of surface markers CD44, CD25, c-Kit or lineage markers (data not shown). Unsupervised k-means clustering of the 24 samples identified five putative gene-set clusters that were associated with stimulation by DII4 (cluster 1), II7 (cluster 2), Dll4+Scf (cluster 3) or DII4+II7+Scf (clusters 4 and 5; Figure 2a; Supplementary Table 1).

a

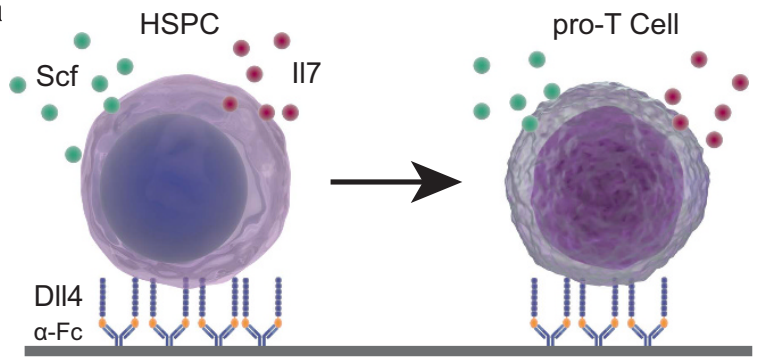

b

Week 1
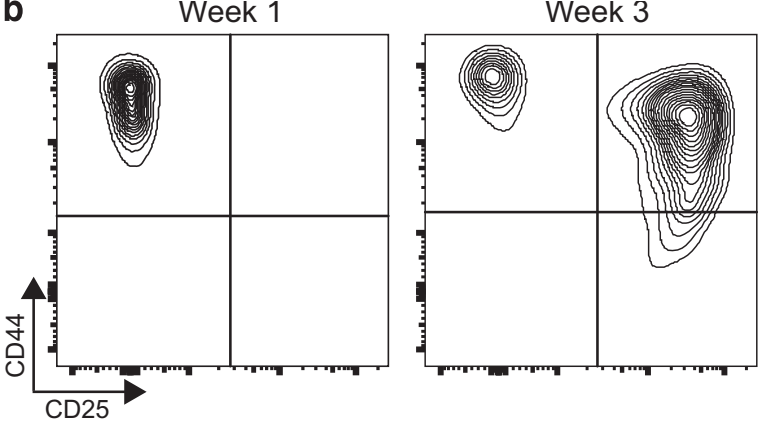

Week 5
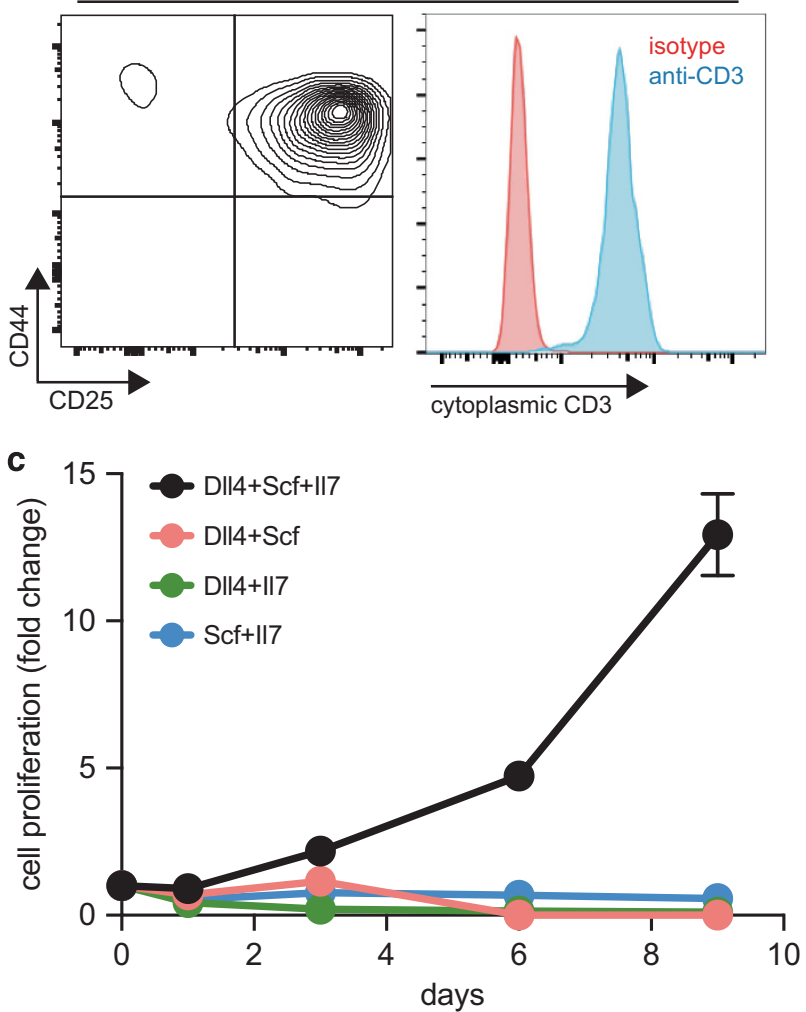

Figure 1. Ex vivo pro-T-cell culture model. (a) Schematic illustrating the differentiation of hematopoietic stem and progenitor cells (HSPCs) into pro-T cells after 5 weeks incubation with Scf, II7 and DII4. (b) Fluorescence-activated cell sorting plots of CD44/CD25 cell surface expression and cytoplasmic CD3 staining during the differentiation into pro-T cells from lineage-negative HSPCs over 5 weeks. (c) Growth curve of pro-T cells in the absence and/or presence of Scf, II7 or DII4. 
Cluster 1 contained a strong Notch target gene signature with upregulation of Hes1, Hey1, Dtx1 and Notch1/3. In silico analysis using ' $\mathrm{i}$-cisTarget' to predict cis-regulatory elements ${ }^{13}$ confirmed enrichment for Rbpj and Runx1 motifs in the promoters of the genes within this cluster (Supplementary Figure 3). These genes were specifically upregulated after the stimulation with DII4 and downregulated when Dll4 was removed from the culture conditions (Figure 2a; Supplementary Figure 4A). Gene ontology analysis showed enrichment for Notch signaling pathways and suppression of pro-B-cell differentiation genes (Supplementary Figure 3).

Cluster 2 identified genes upregulated upon II7 stimulation or downregulated when grown in the absence of II7 (Figure 2a).
These genes showed enrichment for Stat $3 / 5$ cis-regulatory elements using 'i-cisTarget' (Supplementary Figure 3). This cluster included upregulation of known Stat5 target genes such as Cish, $B c 12, X b p 1$ and Socs2 that were not induced by Scf or DII4 (Supplementary Figures $4 \mathrm{~B}$ and $\mathrm{C}$ ).

Cluster 3 genes were predominantly upregulated after combined stimulation with DIl4 and Scf. Stimulation of pro-T cells with Scf, II7 or DII4 alone had no effect on these genes, while removal of Dll4 or removal of Scf significantly affected these genes (Figure 2a). Gene ontology analysis of cluster 3 revealed enrichment for cell cycle, DNA replication and cell proliferation. In particular, 'i-cisTarget' analysis identified enrichment for binding sites of the E2f-family transcription factors and their negative a
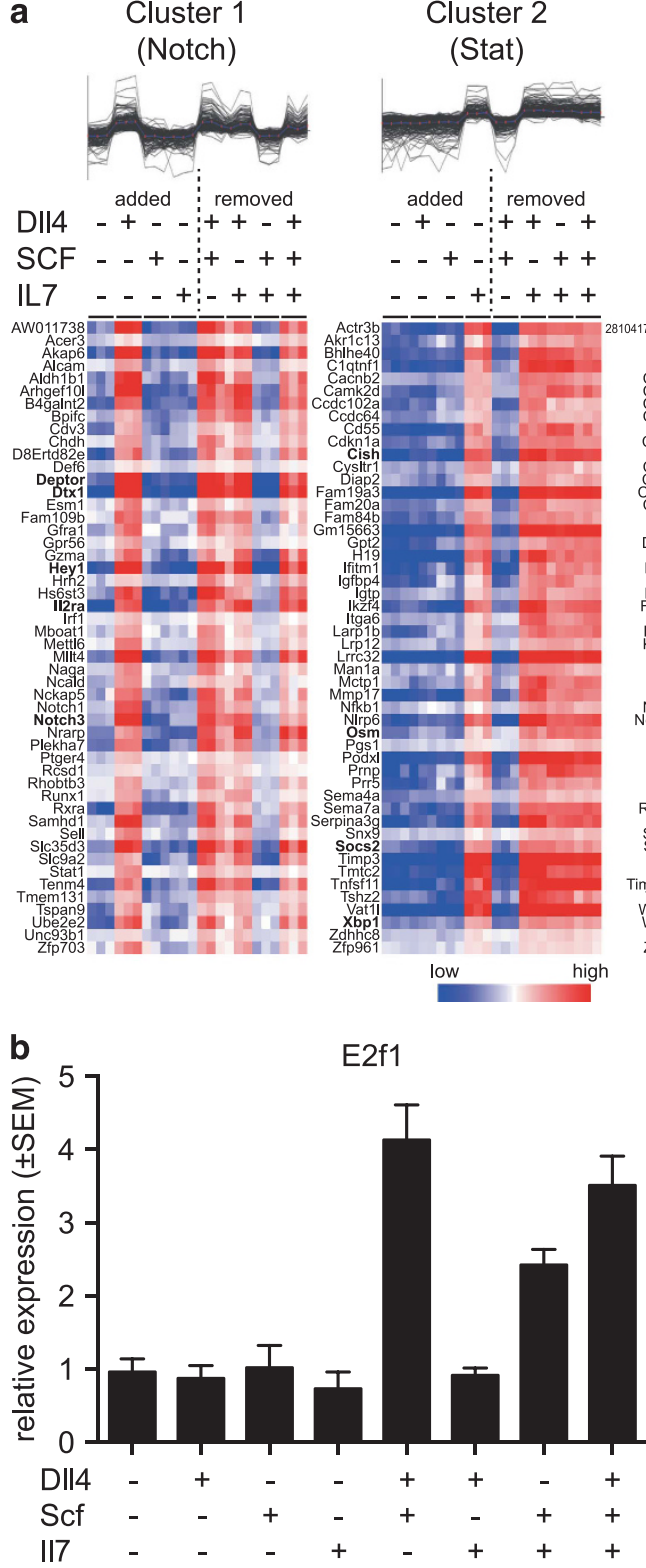

Cluster 3

(E2f)

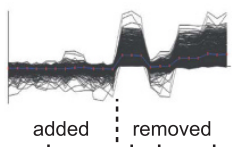

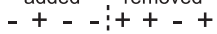

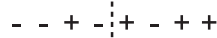

- - + + - ++
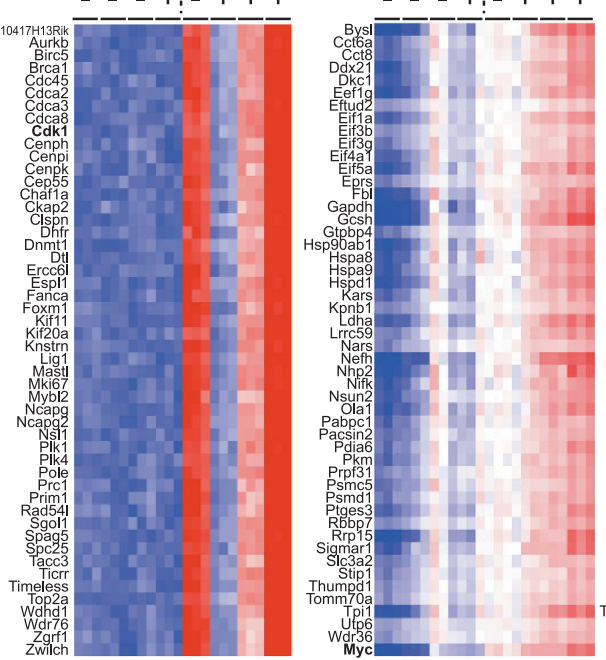

Cluster 4

(Myc)

Cluster 5

(Spi1)

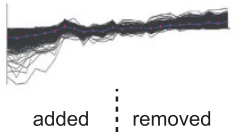

added removed

- + - - + + - +

\begin{tabular}{r:rr}
- & + \\
- & - & + \\
\hline
\end{tabular}

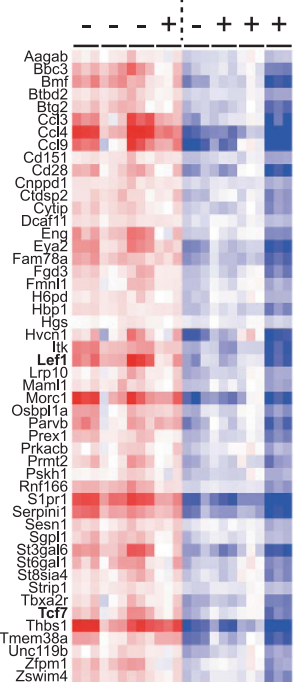


regulator $\mathrm{Rb} 1$ in the promoters of these genes (Supplementary Figure 3). Indeed, E2f1, E2f2 and E2f8 transcript levels remained high in the absence of II7 but decreased when either Dll4 or Scf was removed from the culture conditions (Figure 2b; Supplementary Figure 4D).

The last two clusters were genes either up (cluster 4)- or down (cluster 5)-regulated by all three factors in an additive manner (Figure 2a). Gene ontology analysis of cluster 4 genes revealed signatures for metabolic processes, translation and ribosome biogenesis, and showed enrichment for Myc and Max cisregulatory elements (Supplementary Figure 3). Indeed, Myc expression increased after stimulation with Scf, II7 or DII4, and decreased when each was removed. The highest expression occurred in the presence of DII4, Scf and II7 together (Figure 2c). Conversely, cluster 5 identified genes upregulated in the absence of cytokine and ligand stimulation, and showed enrichment for Spi1 target genes (Figure 2a; Supplementary Figure 3). Spi1 has previously been reported to drive the DN1 transcriptional program during T-cell development and silenced upon further differentiation, ${ }^{14}$ and therefore in part reconciles with their potential repression within the DN2 stage of pro-T cells.

Complementary to this ex vivo analysis, publically available RNAseq data from the different stages of mouse T-cell development were then compared to the pro-T cells and confirmed they represented a bona-fide DN subset (Supplementary Figure 4E). Moreover, the core genes representing each of the defined clusters were also found to be co-regulated across T-cell development (Supplementary Figure 4F). Taken together, these data show that ex vivo cultured pro-T cells have an expression profile representing a bona-fide DN2-DN3 signature and that the identified gene clusters can be linked with Notch1, II7 and/or Scf signaling, and remain co-regulated throughout T-cell development.

Scf and II7 stimulation of pro-T cells results in mutually exclusive activation of Akt and Stat5, respectively

To complement the RNA-seq analysis, phospho-flow cytometry was performed to further dissect the activation of signaling pathways downstream of II7 and Scf. Resting pro-T cells were stimulated with II7 or Scf, and phosphorylation of Stat $5 /$ Stat3 and Akt/mTor was assessed over time (Figures $3 a$ and b). Stimulation with II7 induced rapid phosphorylation of Stat5 peaking within 15 min before rapidly declining (Figures $3 a$ and $c$ ). There was a modest increase in phospho-Stat3 levels following 117 stimulation (Figures $3 \mathrm{c}$ and d). However, II7 was unable to induce Akt or mTor phosphorylation (Figures 3b, e and f). In contrast, stimulation with Scf resulted in the rapid Akt and mTor phosphorylation (Figures $3 b$, e and f), but did not result in any detectable Stat3 or Stat5 phosphorylation (Figures $3 a, c$ and d). Furthermore, there was no significant additive increase when both cytokines were added simultaneously with Scf having only a minor influence on II7-mediated STAT5 phophorylation suggesting that overall the pro-T cells in the DN2 stage have largely exclusive activation of these pathways.

T-ALL-associated mutations confer ligand-independent growth to pro-T cells

Mutations in the NOTCH1, IL7R-JAK-STAT or PI3K-AKT signaling pathways are frequently found in T-ALL patients. ${ }^{3,4,15,16}$ We therefore assessed whether Notch, STAT5, AKT and KIT mutations could confer ligand-independent growth to pro-T cells (Figure 4a). Three different activating Notch 1 mutations (Notch $1^{\text {L1601P }}-\Delta$ Pest, Notch1-ICN and Notch1-ICN- $\triangle$ Pest) were found to confer Dll4-independent growth and shifted the cells toward DN3 phenotype (Figure 4a; Supplementary Figure 5). Notch1-ICN proT-cell proliferation remained dependent on Scf and II7 making this an attractive model to study T-ALL mutations that allow Scf- or II7independent cell growth (Supplementary Figure 6A).
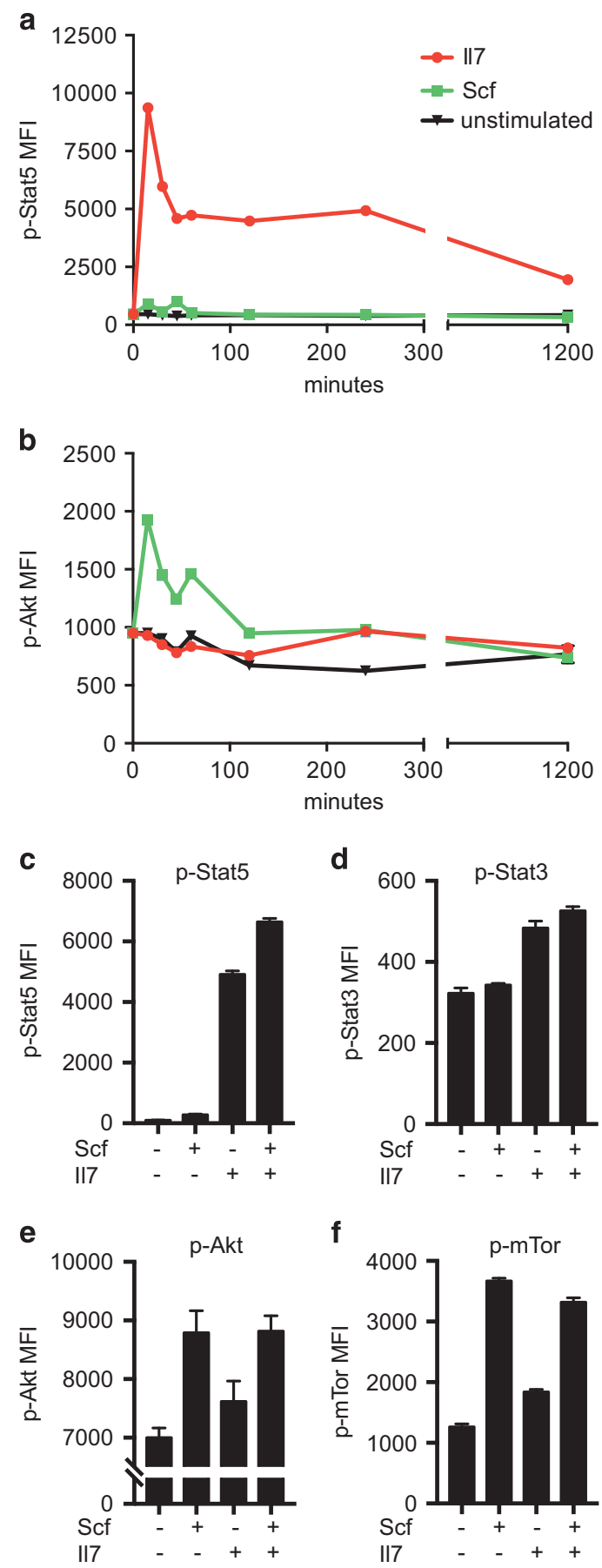

Figure 3. Mutually exclusive activation of Stat5 and Akt by II7 and Scf, respectively. (a) Phospho-flow cytometry time course of $\mathrm{p}$-Stat5 or (b) $\mathrm{p}$-Akt in pro-T cells after $18 \mathrm{~h}$ starvation and then stimulated with either II7 or Scf. (c) p-Stat5, (d) p-Stat3, (e) p-AKT and (f) p-mTor levels assessed by phosphor-flow cytometry in resting pro-T cells and after $15 \mathrm{~min}$ of cytokine stimulation.

Our RNA-seq and phospho-flow cytometry results indicated that activation of Stat5 was only induced by II7 and activation of Akt was only induced by Scf with limited cross talk between these two pathways within this defined setting of pro-T cells at the DN2 stage of differentiation. Therefore, we hypothesized that oncogenic mutations within these pathways would be able to substitute for either 117 or Scf stimulation. Indeed, the activating 
a

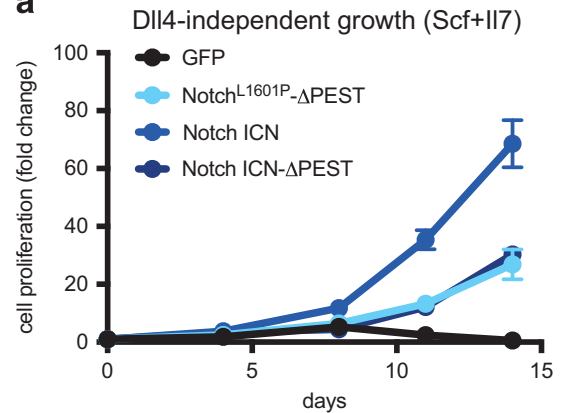

b

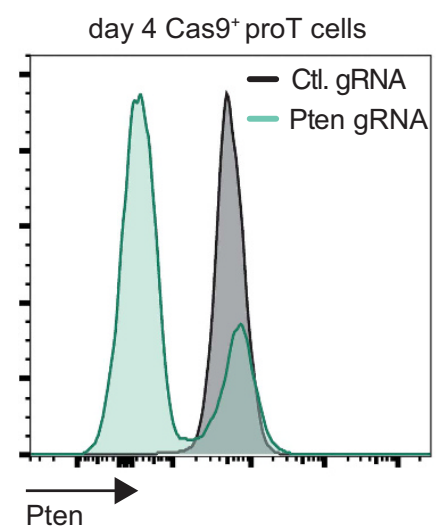

II7-independent growth (DII4+Scf)

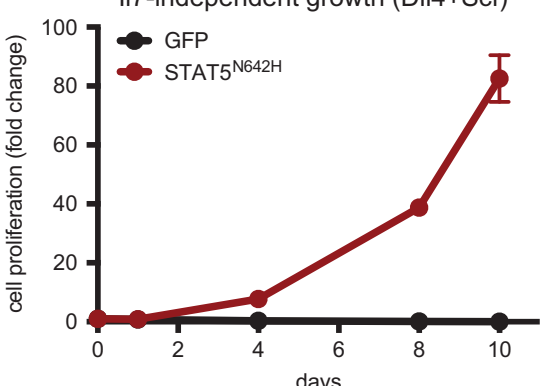

days

Scf-independent growth (DII4+II7)

Cas $9^{+}$proT cells

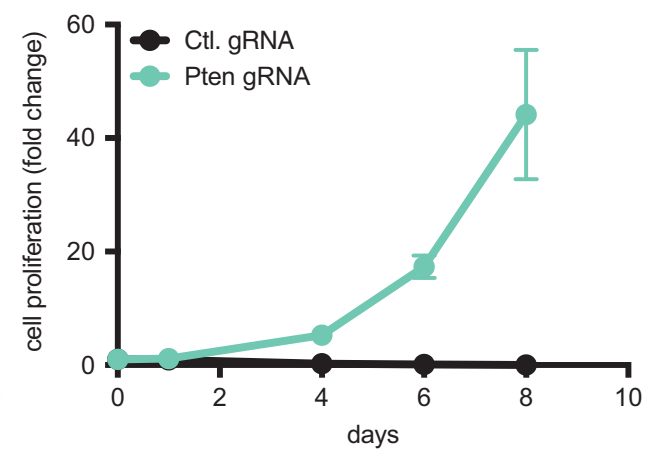

Scf-independent growth (D\|I4+II7)

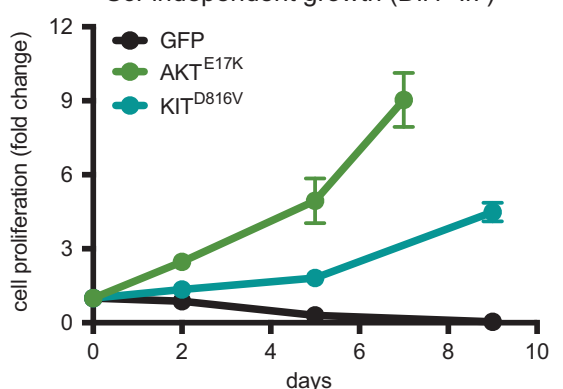

c

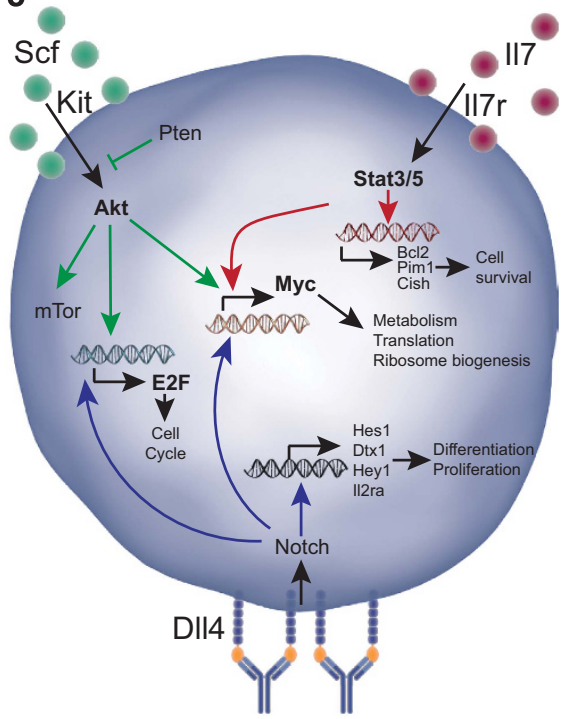

Figure 4. Cytokine-independent growth of pro-T cells driven by oncogene expression. (a) Growth curve of pro-T cells transduced with three different Notch 1 mutations, activating STAT5 ${ }^{\mathrm{N} 542 \mathrm{H}}$ mutation or activating AKT ${ }^{\mathrm{E} 17 \mathrm{~K}}$ and $\mathrm{KIT}^{\mathrm{D} 816 \mathrm{~V}}$ mutations. (b) Intracellular flow cytometry analysis of Pten protein levels in Cas9+ pro-T cells transduced with a negative control guide RNA (Ctl. gRNA) or Pten guide RNA (Pten gRNA), and the growth curve for these cells. (c) Schematic summarizing the main cellular signaling pathways and genes activated after cytokine and Notch-DII4 stimulation in pro-T cells.

STAT5 ${ }^{\mathrm{N} 642 \mathrm{H}}$ mutation conferred II7-independent growth but the cells remained Scf-dependent and remained at the DN2 stage (Figure 4a; Supplementary Figures 5 and 6B). Vice versa, the constitutively active $\mathrm{KIT}^{\mathrm{D} 816 \mathrm{~V}}$ or $\mathrm{AKT}^{\mathrm{E} 17 \mathrm{~K}}$ mutations or inactivation of Pten, using CRISPR/Cas9 technology, conferred Scf-independent growth to the cells, while they remained dependent on 117 (Figures $4 \mathrm{a}$ and $\mathrm{b}$; Supplementary Figure 6C). Expression of $\mathrm{AKT}^{\mathrm{E} 17 \mathrm{~K}}$ also led to the downregulation of CD25 expression reminiscent of a DN1-like phenotype (Supplementary Figure 5).

These data taken together provided a detailed system framework defining the pathways driving pro-T-cell survival and proliferation (Figure 4c). Moreover, this system approach illustrated how specific T-ALL mutations substitute for DII4, II7 and Scf ligands for signaling pathway activation at the DN2-DN3 stage.

TAL1 expression cooperates with PTEN deletion during T-ALL development and silences II7 signaling

T-ALL development is caused by the accumulation of multiple genetic alterations, including the ectopic expression of transcription factors. To determine the interaction between transcription factors and signaling mutations, we used the pro-T-cell system to study the TAL1 transcription factor. TAL1 is a basic helix-loophelix transcription factor and ectopically expressed in $20-30 \%$ of
T-ALL patients either via chromosomal rearrangements ${ }^{3,17}$ or by upstream somatic mutations leading to the generation of a superenhancer. ${ }^{18}$ Analysis of T-ALL sequencing data ${ }^{19}$ revealed a significant correlation between TAL1 expression and PTEN deletion (Figure 5a). This associated between PTEN deletion and TAL1 expression has been reported in a number of previous studies. $^{16,20-22}$ Importantly, our analysis indicated that IL7R-JAK3STAT5-RAS mutations were negatively correlated with TAL1 expression (Figure $5 \mathrm{a}$ ).

Interestingly, expression of TAL1 alone in pro-T cells led to a selective growth disadvantage with the percentage of $\mathrm{TAL}^{+}$ $\left(\mathrm{GFP}^{+}\right.$) pro-T cells decreasing over time (Figure 5b). On the basis of the mutations found in TAL1-positive T-ALL cases, such as the prevalence for PTEN deletions, we hypothesized that strong AKT activation could be required to drive the proliferation of $\mathrm{TAL} 1^{+}$ cells. Indeed, expression of an AKT ${ }^{\mathrm{E} 17 \mathrm{~K}}$-activating mutation or inactivation of Pten rescued the TAL1-induced growth disadvantage, with the Pten ${ }^{\text {del. }} / \mathrm{TAL}^{+}{ }^{+}$or $\mathrm{AKT}^{\mathrm{E} 17 \mathrm{~K}} / \mathrm{TAL} 1^{+} \mathrm{DP}$ pro- $\mathrm{T}$ cells becoming the major clone in the presence of DII4, Scf and II7 (Figure 5c; Supplementary Figure 7A). In contrast, other oncogenic mutations such as KRAS ${ }^{\mathrm{G} 12 \mathrm{D}}$ or JAK3 ${ }^{\mathrm{M} 5111}$ were unable to induce expansion of TAL1-expressing cells (Figure $5 c$ ).

To dissect the transcriptional network reprogramming underlying the cooperation between TAL1 expression and Pten deletion, 
a

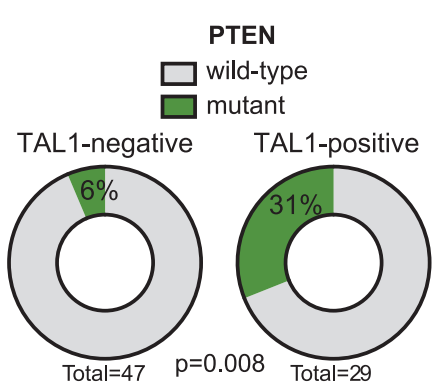

b

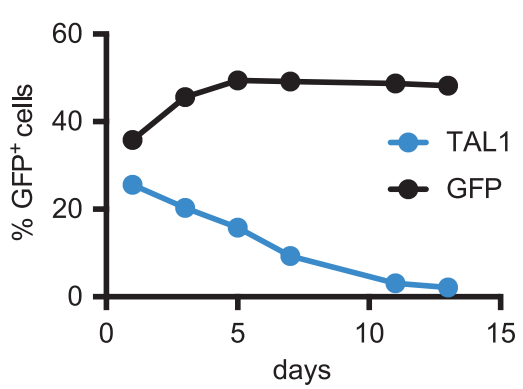

C

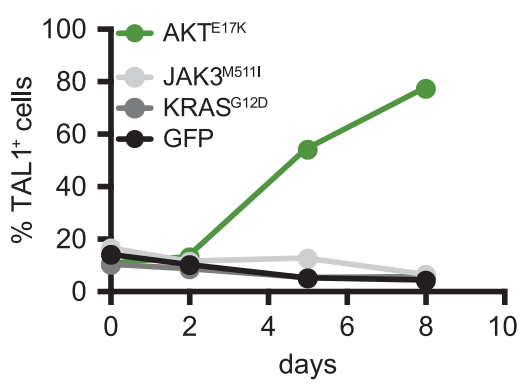

d

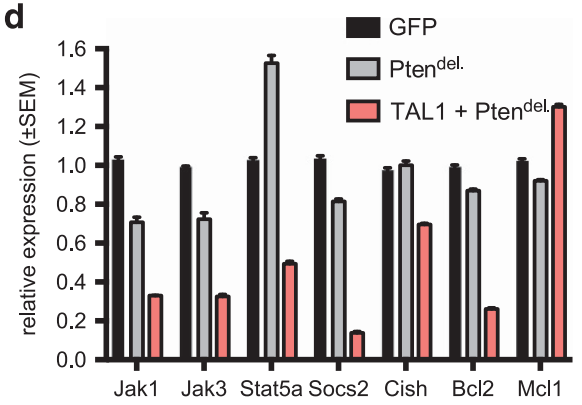

f

\section{IL7R-JAK3 \\ STAT5-RAS \\ $\square$ wild-type \\ $\square$ mutant}
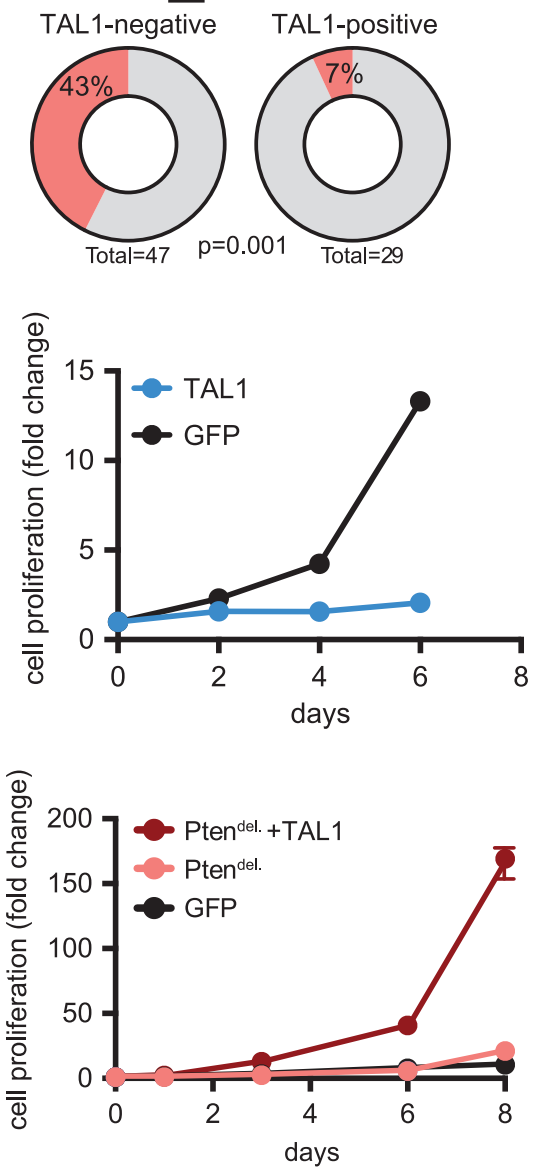

e

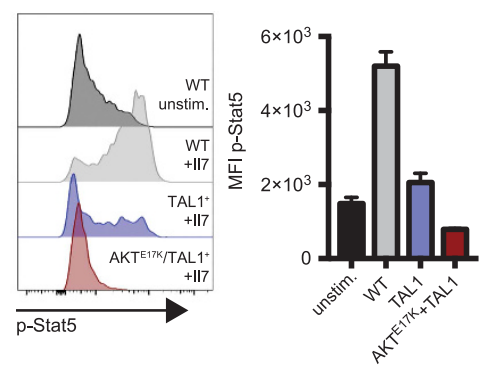

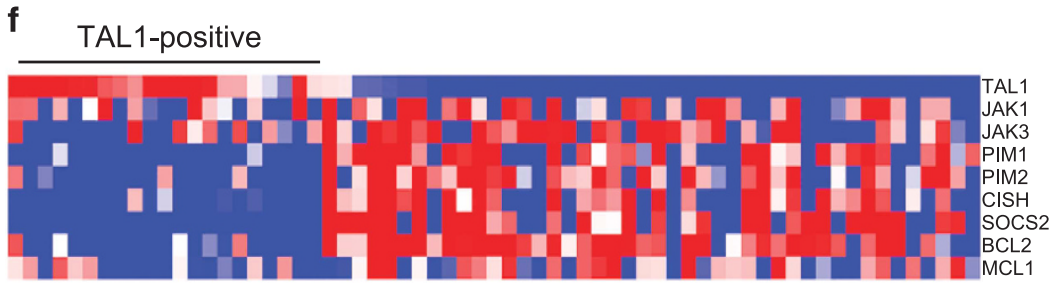

Figure 5. Loss of PTEN and expression of TAL1 cooperate in driving cytokine-independent growth of pro-T cells. (a) Analysis of clinical T-ALL patient data correlating TAL1 expression with loss of PTEN and correlating TAL1 expression with mutations in the IL7-JAK-STAT or RAS signaling pathways. (P-values calculated using the Pearson's $X^{2}$-test.) (b) In vitro competitive growth assessment between pro-T cells transduced with TAL1-IRES-GFP or GFP only. (c) Growth curve of TAL1-IRES-mCherry pro-T cells co-transduced with either activating AKT ${ }^{\text {E17K }}$ -

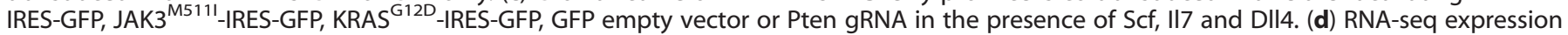
data for JAK-STAT signaling proteins and known Stat5 target genes. (e) Phospho-flow cytometry analysis of p-Stat5 levels in non-transduced pro-T cells (wild type; WT), TAL1 and AKT ${ }^{\mathrm{E} 17 \mathrm{~K}} / \mathrm{TAL} 1+$ cells after 15 min II7 stimulation. (f) Heat map of T-ALL patient samples clustered by TAL1 expression and assessed for expression of canonical IL7-JAK-STAT target genes. 
RNA sequencing was performed on unstimulated GFP ${ }^{+}$, Pten ${ }^{\text {del. }}$ and Pten ${ }^{\text {del. }} / \mathrm{TAL}^{+}$pro-T cells. Gene ontology analysis of genes differentially expressed between Pten ${ }^{\text {del. }} / \mathrm{TAL} 1^{+}$and Pten ${ }^{\text {del. }}$ pro-T cells revealed a negative enrichment for JAK-STAT signaling cascade (Supplementary Figure 7B), a concomitant decrease in genes directly involved in this pathway including Jak1, Jak3, Stat3 and Stat5, and a decrease in canonical Stat5 target genes such as Cish, Socs2 and Bcl2 (Figure 5d). Moreover, phospho-flow cytometry analysis showed that $\mathrm{TAL}^{+}{ }^{+}$and $\mathrm{AKT}{ }^{\mathrm{E} 17 \mathrm{~K}} / \mathrm{TAL} 1^{+}$pro-T cells were unable to induce Stat5 phosphorylation upon II7 stimulation (Figure 5e).

This finding that TAL1 expression is associated with reduced IL7R-JAK-STAT signaling was also corroborated within TAL1positive T-ALL patient samples (Figure 5f; Supplementary Figures 7C and D). Furthermore, publically available TAL1 ChIP-seq in CCRF-CEM and Jurkat cells revealed TAL1 bound directly downstream of the IL7R genomic region suggesting direct gene repression (Supplementary Figure $7 \mathrm{E}$ ). ${ }^{23}$ These data demonstrate that $48 \mathrm{~h}$ of TAL1 expression leads to reduced 117 responsiveness and in part may explain the lack of expansion of $\mathrm{TAL}^{+}$pro-T cells in the presence of 117 and the absence of IL7R/JAK/STAT pathway mutations in TAL1-positive T-ALL patients.

In agreement with the finding that $\mathrm{TAL}^{+}$cells were II7 hypo-responsive, we found that Pten ${ }^{\text {del. }} / \mathrm{TAL}^{+}{ }^{+}$pro-T cells could grow in the absence of 117 , in contrast to Pten ${ }^{\text {del. }}$ or $\mathrm{GFP}^{+}$cells (Figure 6a). Somewhat surprisingly, Pten ${ }^{\text {del. }} / \mathrm{TAL}^{+}{ }^{+}$pro-T cells also became Dll4-independent and were able to grow in the presence of only Scf (Figure 6a). The same was true for AKT-activating mutation in combination with TAL1 expression (Supplementary Figure 7F). These data suggest that TAL1 may reprogram the transcriptional network to alter the response of pro-T cells to external signals, repressing II7-mediated signaling and inducing Scf dependency.

To determine the mechanism and signaling network responsible for the expansion of Pten ${ }^{\text {del. }} / \mathrm{TAL}^{+}$cells in the absence of IL7R/JAK/STAT signaling, differential expression analysis of Scfstimulated versus -unstimulated Pten ${ }^{\text {del. }} / \mathrm{TAL}^{+}{ }^{+}$cells was undertaken. This analysis revealed that Scf induced minor changes in gene expression, with a skewed upregulation of genes, many of which were part of the previously defined Myc cluster (Supplementary Figures $8 \mathrm{~A}-\mathrm{C}$ ). The previous gene ontology analysis showed strong positive upregulation for genes involved in cell proliferation (Supplementary Figure 7B). Indeed, Pten ${ }^{\text {del. }}$ pro-T cells and also Pten ${ }^{\text {del. }} /$ TAL $^{+}{ }^{+}$cells showed significant enrichment for the previously defined E2f cluster gene set (Supplementary Figures $9 \mathrm{~A}$ and B). Pten deletion alone upregulated expression of the transcription factors E2f1, E2f2 and E2f8, and their target genes, and was further increased by TAL1 (Figure 6b). Interestingly, publically available ChIP-seq data for Jurkat and CCRF-CEM cells revealed TAL1 directly bound to E2F1 and E2F2 promoter/enhancer regions (Supplementary Figure 9CD). Furthermore, the top-ranked E2F cluster genes, and E2F1, E2F2 and E2F8 transcription factors are higher in $\mathrm{TAL} 1^{+} \mathrm{T}-\mathrm{ALL}$ patient samples compared to $\mathrm{HOX}^{+}$or immature T-ALL samples (Figure 6c; Supplementary Figures $9 \mathrm{E}$ and F).

In addition to the negative association with the JAK-STAT signaling cascade in Pten ${ }^{\text {del. }} / \mathrm{TAL}^{+}$pro-T cells, there was significant downregulation of gene sets involved T-cell differentiation and T-cell receptor recombination (Supplementary Figure 7B). Detailed analysis revealed increased expression of the stem cell marker CD34 (Figure 7a) and decreased expression of T-cell commitment transcription factors (Figure 7b). We therefore hypothesized that this immature T-cell phenotype could be part of a stem cell-like gene expression program, driven by TAL1 as previously reported within a TAL1/LMO1 transgenic mouse model. ${ }^{24}$ Indeed, gene-set enrichment analysis confirmed that genes upregulated by Pten ${ }^{\text {del. }} / T A L 1^{+}$were also significantly enriched in genes upregulated in hematopoietic stem cells, and
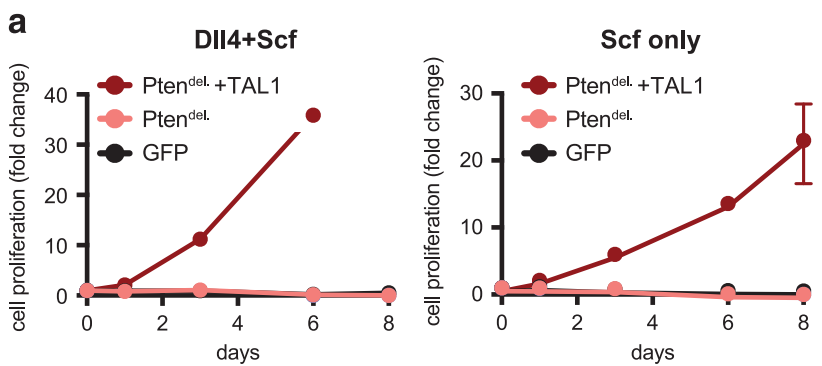

b
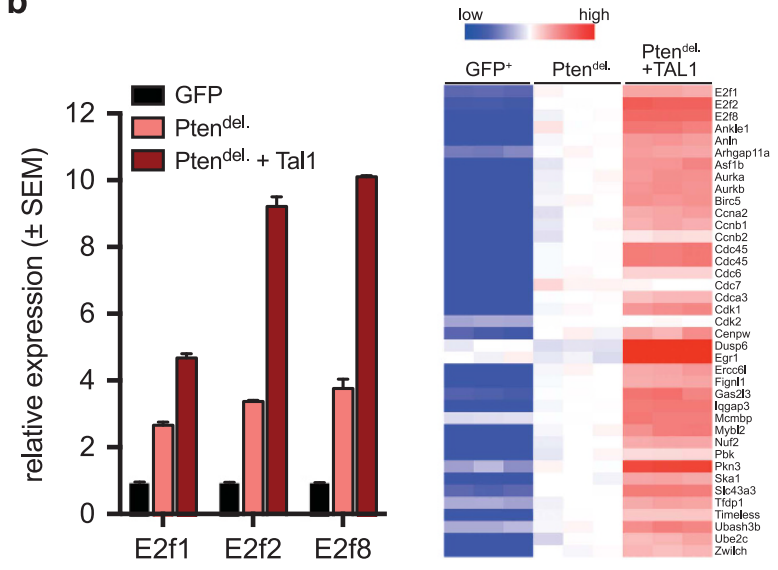

C
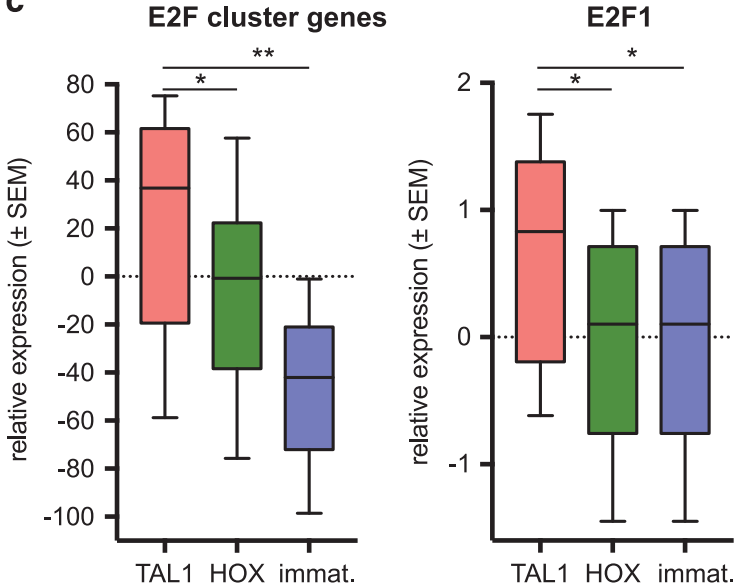

Figure 6. Pten ${ }^{\text {del. }} / \mathrm{TAL}^{+}{ }^{+}$pro-T cells become $\| 17$-independent and cooperatively activate the E2f transcriptional network. (a) Growth curve of Pten ${ }^{\text {del. }}$, Pten ${ }^{\text {del. }}$ /TAL1 and GFP pro-T cells in the absence of II7 stimulation, or absence of DII4 and II7. (b) Expression analysis of E2f1, E2f2 and E2f8 transcription factors with a heat map of the top 40 E2f cluster genes in pro-T cells. (c) The sum expression of the homologous human E2F cluster genes in TAL1, HOX and immature T-ALL patients and relative expression level of E2F1 transcription factor in the same T-ALL patient samples. Significance was calculated using Student's $t$-test $\left({ }^{*} P \leqslant 0.01 ;{ }^{*} P \leqslant 0.05\right)$.

vice versa, genes downregulated by $\mathrm{Pten}^{\text {del. }} / \mathrm{TAL}^{+}{ }^{+}$were also negatively enriched in genes downregulated by leukemic stem cells $^{25,26}$ (Figure 7c). This Pten ${ }^{\text {del. }} / \mathrm{TAL}^{+}$-induced reprogramming also increased Lmo1, Lmo2 and Spi1 expression, and therefore reconciles in part with 'self-renewal reprogramming' found in transgenic mice ${ }^{24}$ (Figure 7d).

The phenotype of TAL1-positive patients has previously been described as more mature and CD4/CD8 DP. ${ }^{27}$ However, in line 
a

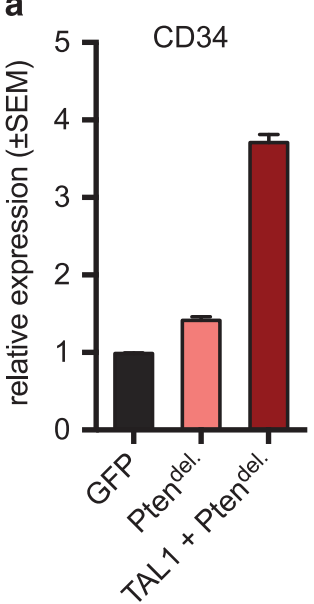

C

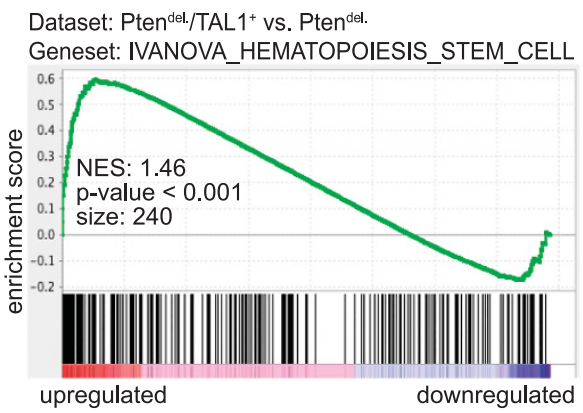

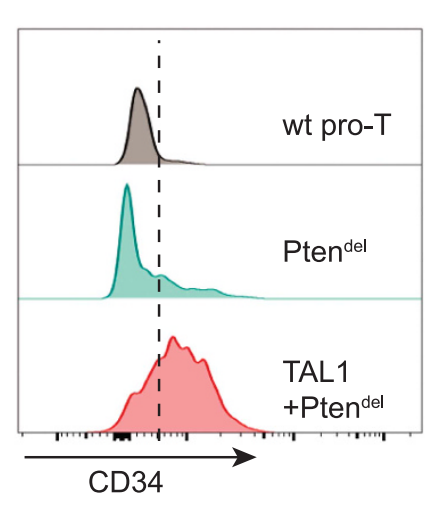

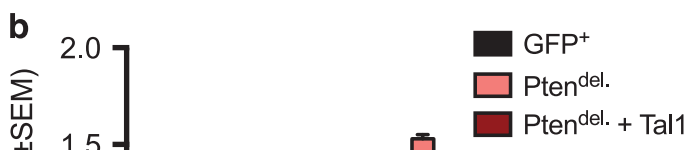

e

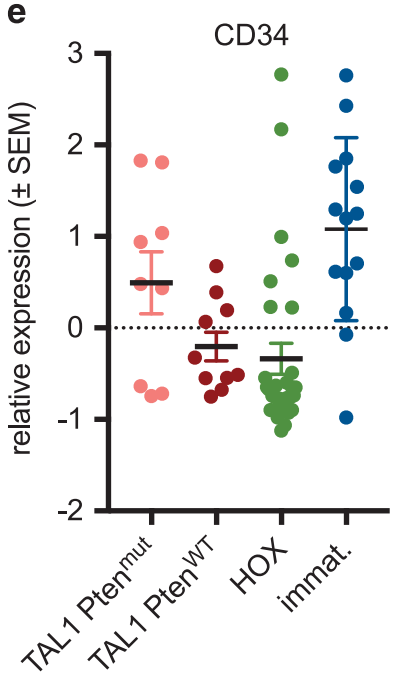

Dataset: Pten ${ }^{\text {del }} /$ TAL $1^{+}$vs. Pten ${ }^{\text {del. }}$

Geneset: GAL_LEUKEMIC_STEM_CELL_down

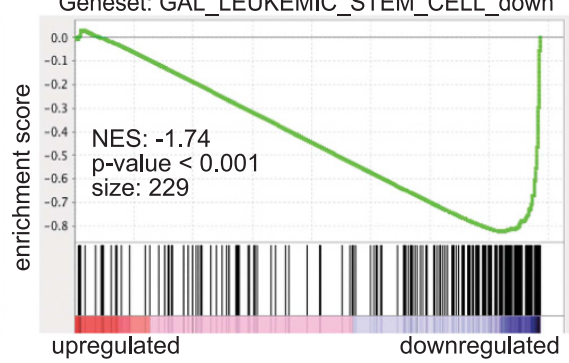

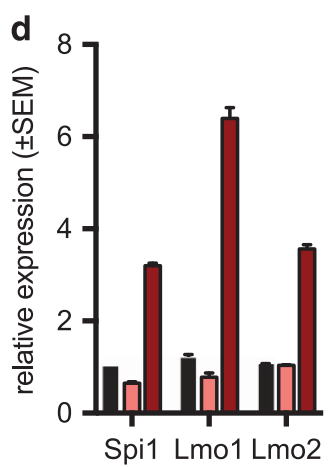
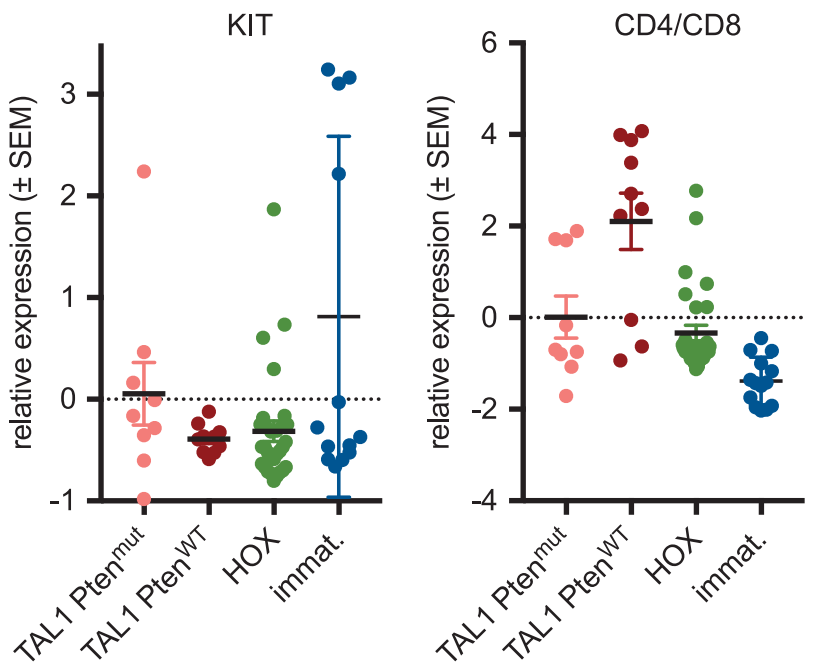

Figure 7. Pten ${ }^{\text {del. }} / \mathrm{TAL}^{+}$pro-T cells drive T-cell de-differentiation and induce a stem cell-like phenotype. (a) Expression levels of CD34 in transduced pro-T cells from RNA-seq and flow cytometry analysis. (b) RNA-seq expression analysis of T-cell differentiation transcription factors. (c) Gene set enrichment analysis of differentially expressed genes between Pten ${ }^{\text {del. }} / \mathrm{TAL}^{+}{ }^{+}$versus Pten ${ }^{\text {del. }}$ pro-T cells against a stem cell signature ${ }^{25}$ and genes downregulated in leukemic stem cells. ${ }^{26}$ (d) RNA-seq expression analysis stem cell transcription factors. (e) Expression of CD34, KIT and CD4/CD8 in subgroups of T-ALL patient samples.

with our observation, TAL1-positive T-ALL patients with PTEN deletion were found to express CD34 and the KIT receptor at higher levels, whilst markers of T-cell differentiation (CD4 and CD8) were at lower levels compared to TAL1-positive T-ALL patients without PTEN deletion (Figure 7e). The TAL1-positive with Notch mutations were CD4+/CD8+ as previously described (Figure 7e).
TAL1 expression drives metabolic reprogramming of pro-T cells to increase expression of lactate transporters

The gene ontology analysis of Pten ${ }^{\text {del. }} / \mathrm{TAL}^{+}{ }^{+}$pro-T cells showed positive enrichment for metabolic processes and carbohydrate metabolic processes (Supplementary Figure 7B). Therefore, we investigated whether we could detect changes in glucose metabolism within the pro-T cells. To this end, GFP ${ }^{+}$, Pten $^{\text {del. }}$ 
and Pten ${ }^{\text {del. }} / \mathrm{TAL}^{+}{ }^{+}$cells were cultured for $24 \mathrm{~h}$ in the presence of ${ }^{13} \mathrm{C}$-labeled glucose. Mass spectrometry analysis of metabolites and their fractional labeling revealed a significant increase in labeling of the tricarboxylic acid cycle intermediates, such as citrate, malate and fumarate, in $\mathrm{Pten}^{\text {del. }} / \mathrm{TAL}^{+}$pro-T cells compared to $\mathrm{GFP}^{+}$or Pten ${ }^{\text {del. }}$ cells (Supplementary Figure 10).
We next determined whether the rapidly dividing Pten ${ }^{\text {del. }}$ $\mathrm{TAL}^{+}$pro-T cells might have higher lactate levels due to the increased flux of glucose into the tricarboxylic acid cycle to support cell growth. In line with previous research, loss of Pten resulted in increased lactate labeling, and intracellular lactate accumulation and was equivalent in TAL1/Pten ${ }^{\text {del. }}$ cells
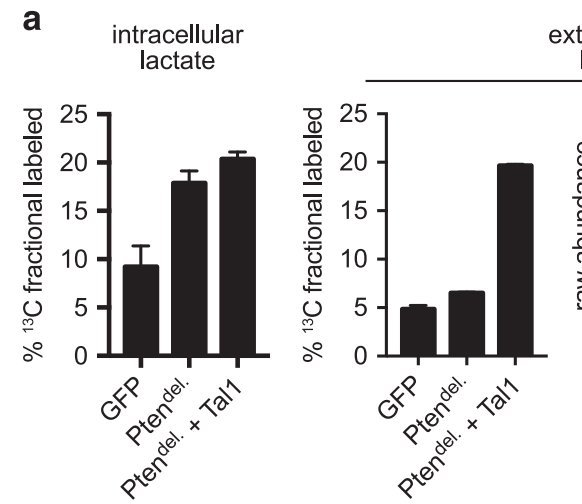

extracellular lactate
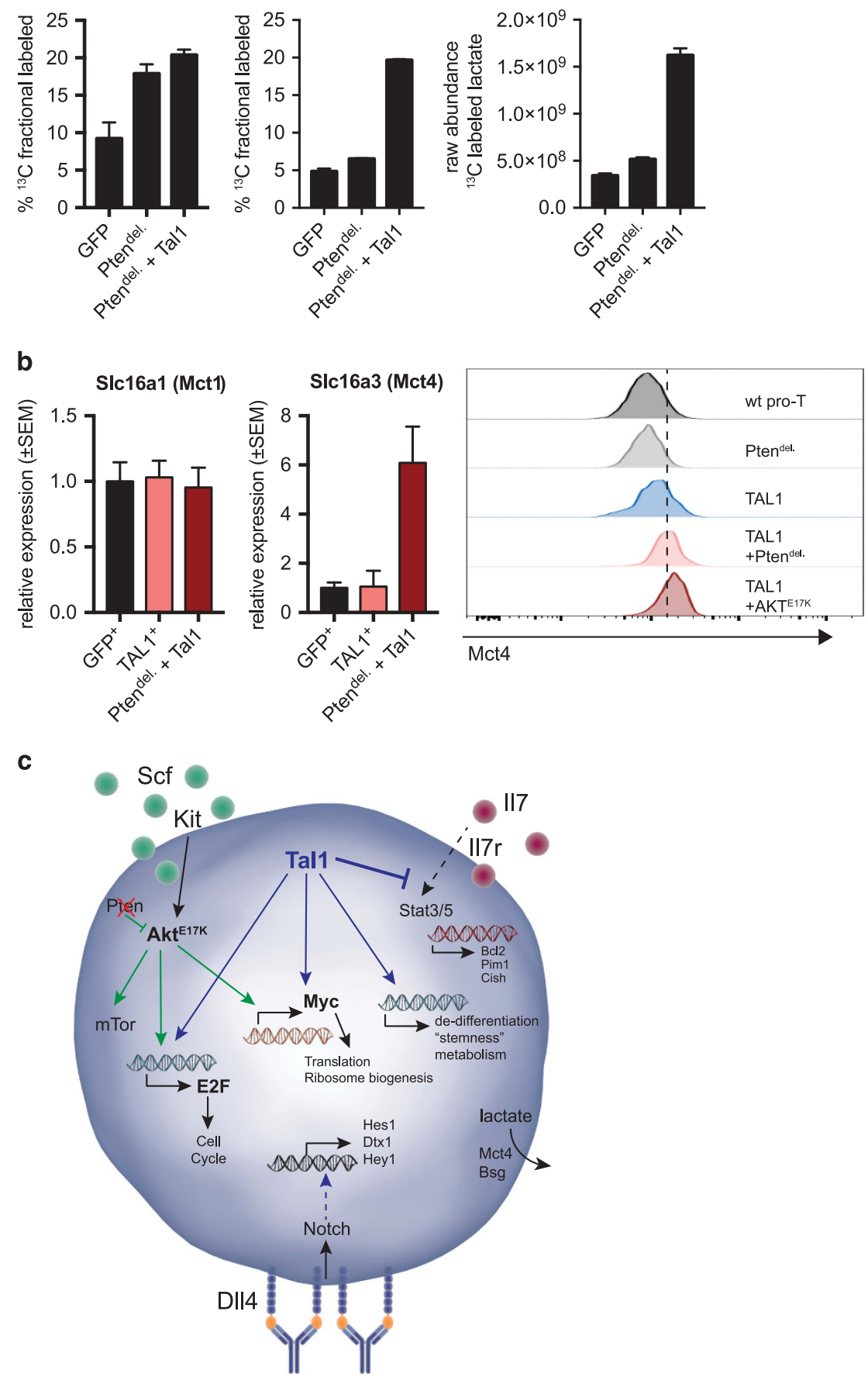

Figure 8. Pten ${ }^{\text {del. }} / \mathrm{TAL}^{+}$cells increase tricarboxylic acid metabolism and upregulate the lactate export protein Mct4. (a) Percentage of intracellular and extracellular ${ }^{13} \mathrm{C}$-labeled lactate as measured by liquid chromatography-mass spectrometry. (b) Real-time PCR analysis of RNA expression of Slc16a1 (Mct1) and Slc16a3 (Mct4), and flow cytometry analysis of Mct4 protein expression in pro-T cells. (c) Schematic summarizing the signaling network in pro-T cells and their relationship with the mutations found in Akt, deletion of Pten and Tal1 overexpression. 
(Figure $8 a) .^{28}$ Interestingly, TAL1/Pten ${ }^{\text {del. }}$ cells had significantly higher levels of extracellular lactate compared to Pten ${ }^{\text {del. }}$ cells (Figure $8 \mathrm{a}$ ) suggesting that $\mathrm{TAL}^{+}$cells might increase expression of lactate transporters to circumvent intracellular accumulation of potential cytotoxic lactate. Indeed, the expression of the lactate exporter Slc16a3 (Mct4) was significantly increased in Pten del./ $\mathrm{TAL}^{+}$pro-T cells (Figure $8 \mathrm{~b}$ ). Also the Mct4 protein was highly expressed on Pten ${ }^{\text {del. }} / \mathrm{TAL}^{+}$and $\mathrm{AKT}^{\mathrm{E} 17 \mathrm{~K}+} / \mathrm{TAL} 1^{+}$pro-T cells, whereas TAL1 expression or Pten deletion alone did not affect expression (Figure $8 \mathrm{~b}$ ). In summary, these data now provide a model of TAL1-mediated T-cell transformation in the context of Pten deletions (Figure 8c).

\section{DISCUSSION}

Recent discoveries regarding the genetic basis of T-ALL have provided new insight in the development of this malignancy and we now have a good overview of the various pathways that are altered in T-ALL. ${ }^{3,29}$ However, many of the in vitro model systems used to study developmental and oncogenic T-cell signaling pathways ex vivo can be limiting due to either not being physiological or require the use of feeder-cell lines. Here we analyzed the pro-T-cell culture system as a new cellular model and show using a systems biology approach that pro-T cells recapitulate thymic $T$ cell at the DN2-DN3 stage. We also identified distinct transcriptional networks in the pro-T cells downstream of II7, Scf and DII4. Significantly, the pro-T-cell transcriptome clusters reconciled with the in vivo DN2-DN3 T-cell stages and revealed somewhat surprisingly that there was limited cross talk between the $\mathrm{II} \mathrm{r}$ and PI3k/Akt signaling with no Akt phosphorylation observed in response to II7 stimulation and vice versa no Stat5 phosphorylation in response to Scf stimulation.

Indeed, our results suggest that within this very defined culture system of pro-T cells at the DN2 stage of differentiation, each of the external signals largely act independently of one another in activating Notch (downstream of DLL4), p-Akt (downstream of Scf) and p-Stat5 (downstream of II7). Previous reports have shown that IL7R signaling can activate the PI3K/AKT pathway, but in contrast to our study, this was in more mature $T$ lymphocytes ${ }^{30}$ or in the IL7-dependent TAIL7 cell line. ${ }^{31}$ Similarly, cross talk between PI3/ Akt and II7r pathways was observed in a transgenic mouse model where Pten deletion induced by Lck-Cre could substitute for IL7R signaling. ${ }^{32}$ However, Lck-Cre activity begins at the DN3 stage peaking at the CD4/CD8 DP stage, ${ }^{33}$ and recently Lck-Cre expression was found to directly increase the frequency of $\| 7 \mathrm{r}$ thymocytes. ${ }^{34}$ Cross talk has also been reported between Notch signaling and P13K/AKT in both human T-ALL cell lines and DN3 thymocytes on a OP9-DL1 culture system in which Notch represses Pten expression. ${ }^{35}$ We cannot preclude cross talk between these pathways in pro-T-cell DN2 context between upstream members of the pathways (for example, via proteinprotein interactions), but our results as presented here suggest that there is only limited cross talk between the IL7R signaling pathway, NOTCH and PI3K/AKT that ultimately leads to either STAT5 or AKT activation at this stage of is likely to be related to the T-cell maturation stage.

Our analysis here also identified the importance of E2f and Myc gene clusters during T-cell development. The genes within these clusters were co-regulated throughout T-cell development peaking during DN4 to DP transition. Previous reports demonstrated that developing T-cell proliferation is strictly required for DN4-DP transition, ${ }^{36}$ and we present data showing that Myc expression is controlled by multiple inputs, including Stat5, Akt and Notch1 signaling. Furthermore, E2f was controlled by synergistic Notch and Scf-Kit-mediated Akt activation. However, Kit is silenced upon further maturation of T cells, and therefore other receptors such as the pre-T-cell receptor might influence Akt-mediated E2f activation.
Having defined the signaling pathways downstream of Scf, II7 and Notch in pro-T cells, we then sought to understand the relationship between PTEN deletion and TAL1 overexpression, which often co-occurs in T-ALL, ${ }^{19-21}$ but whose molecular mechanism of oncogenic cooperation has remained somewhat unclear. Interestingly, TAL1 expression alone induced a growth disadvantage to pro-T cells, which was relieved specifically by AKT pathway activation. Indeed, our data show that TAL1 silences the IL7R-JAK-STAT signaling cascade and therefore requires activation of AKT to stimulate E2f and Myc expression. This latter point reconciles with TAL1-positive T-ALL cases have a high frequency of AKT/PTEN mutations, and absence of IL7R/JAK/STAT mutations and low expression levels of STAT5 target genes, such as CISH, PIM1 and BCL2. Translating these finding to the clinic, $\mathrm{TAL}^{+}$ patients are therefore unlikely to benefit from therapies targeting the anti-apoptotic protein BCL2 and this was recently confirmed within a patient sample carrying a SIL-TAL fusion that had a very high IC50 for the BCL2 inhibitor ABT199. ${ }^{37}$ Our data do demonstrate that Pten ${ }^{\text {del. }} / \mathrm{TAL}^{+}{ }^{+}$cells maintain MCL1 expression and therefore may be more sensitive to a specific MCL1 inhibitor such as $\mathbf{S 6 3 8 4 5}$ that has shown to have excellent efficacy across a broad range of $\mathrm{MCL} 1$-dependent hematological malignancies. ${ }^{38}$ The Pten ${ }^{\text {del. }} / \mathrm{TAL}^{+}$pro-T cells induced stem cell-like phenotype was also accompanied by metabolic reprogramming of the cells. This resulted in not only increased tricarboxylic acid cycle activity but also increased lactate production, presumably exported outside the cells via the significant increased expression of Mct4 transporter. It is unclear whether TAL1 directly regulates these transporters at the genetic level or are indirect downstream secondary events. Interestingly, metabolic inhibitors are currently under development for multiple types of cancer and therefore these may also be beneficial for Pten ${ }^{\text {del. }} / \mathrm{TAL} 1^{+} \mathrm{T}-\mathrm{ALL}$ patients.

What also became clear is that patients with PTEN ${ }^{\text {del. }} / \mathrm{TAL}^{+}$ cells upregulate a 'stem-like' signature that was distinct from more mature TAL1 ${ }^{+}$that have wild-type PTEN cases but harbor Notch mutations. From a therapeutic perspective, these results suggest that PTEN ${ }^{\text {del. }} / \mathrm{TAL}^{+}{ }^{+}$patients would benefit from inhibition of $\mathrm{c}-\mathrm{KIT}$. Indeed a recent publication identified $\mathrm{TAL}^{+}$patients as particularly sensitive to dasatinib, which is also known to inhibit c-KIT amongst other kinases. ${ }^{39}$ Biologically, the ectopic acquisition of TAL1 expression is known to block cellular differentiation by preventing progression from DN2 to CD4+CD8+ DP stage and thereby remaining in the thymic microenvironment until the acquisition of secondary mutations. ${ }^{40,41}$ Our data suggest that in this scenario, if the secondary hit is deletion of PTEN it could drive de-differentiation via a 'stem-like' program. However, if the secondary hit were the acquisition of a Notch mutation, it could drive a mature cortical $\mathrm{CD} 4^{+} \mathrm{CD} 8^{+} \mathrm{T}$-ALL phenotype.

In conclusion, we have used a systems biological approach to define the signaling pathways driving pro-T-cell proliferation and survival then used this system to dissect the underlying molecular basis in Pten ${ }^{\text {del. }} /$ TAL1 cooperation. This new ex vivo model system is exquisitely placed to understand how different mutations found in T-ALL patients can drive ligand-independent growth and thereby identify new potential targets for therapy.

\section{CONFLICT OF INTEREST}

The authors declare no conflict of interest.

\section{ACKNOWLEDGEMENTS}

This work was supported by grants from KU Leuven (PF/10/016 SymBioSys), FWOVlaanderen, Foundation against Cancer, and by an ERC-consolidator grant (to JC). SB is an Aspirant of FWO-Vlaanderen. 


\section{AUTHOR CONTRIBUTIONS}

$S B, O G, E G, B G, R S$ and $C E d B$ performed experiments. SB analyzed all data with conceptual and intellectual input $S A, J C$ and CEdB. SD contributed to all genomics and bioinformatics analysis. SB, CEdB and JC conceived the study and wrote the manuscript. CEdB and JC jointly supervised this work.

\section{REFERENCES}

1 Van Vlierberghe $\mathrm{P}$, Ferrando A. The molecular basis of $\mathrm{T}$ cell acute lymphoblastic leukemia. J Clin Invest 2012; 122: 3398-3406.

2 Weng AP, Ferrando AA, Lee W, Morris JP, Silverman LB, Sanchez-Irizarry C et al. Activating mutations of NOTCH1 in human T cell acute lymphoblastic leukemia. Science 2004; 306: 269-271.

3 Girardi T, Vicente C, Cools J, De Keersmaecker K. The genetics and molecular biology of T-ALL. Blood 2017; 129: 1113-1123.

4 Van Vlierberghe $P$, Ferrando $A$, van Vlierberghe $P$, Ferrando $A$. The molecular basis of T cell acute lymphoblastic leukemia. J Clin Invest 2012; 122: 3398-3406.

5 Kleppe M, Mentens N, Tousseyn T, Wlodarska I, Cools J. MOHITO, a novel mouse cytokine-dependent T-cell line, enables studies of oncogenic signaling in the T-cell context. Haematologica 2011; 96: 779-783.

6 Holmes R, Zuñiga-Pflücker JC. The OP9-DL1 system: Generation of T-lymphocytes from embryonic or hematopoietic stem cells in vitro. Cold Spring Harb Protoc 2009; 4: pdb.prot5156.

7 Klein L, Kyewski B, Allen PM, Hogquist Ka. Positive and negative selection of the T cell repertoire: what thymocytes see (and don't see). Nat Rev Immunol 2014; 14: 377-391.

8 Radtke F, Wilson A, Stark G, Bauer M, Van Meerwijk J, MacDonald HR et al. Deficient $T$ cell fate specification in mice with an induced inactivation of Notch1. Immunity 1999; 10: 547-558.

9 Han H, Tanigaki K, Yamamoto N, Kuroda K, Yoshimoto M, Nakahata T et al. Inducible gene knockout of transcription factor recombination signal binding protein-J reveals its essential role in T versus B lineage decision. Int Immunol 2002; 14: 637-645.

10 Gehre N, Nusser A, von Muenchow L, Tussiwand R, Engdahl C, Capoferri G et al. A stromal cell free culture system generates mouse pro-T cells that can reconstitute T-cell compartments in vivo. Eur J Immunol 2015; 45: 932-942.

11 Ikawa T, Hirose S, Masuda K, Kakugawa K, Satoh R, Shibano-Satoh A et al. An essential developmental checkpoint for production of the $T$ cell lineage. Science 2010; 329: 93-96.

12 Dagklis A, Demeyer S, De Bie J, Radaelli E, Pauwels D, Degryse S et al. Hedgehog pathway activation in T-cell acute lymphoblastic leukemia predicts response to SMO and GLI1 inhibitors. Blood 2016; 128: 2642-2654.

13 Imrichová H, Hulselmans G, Kalender Atak Z, Potier D, Aerts S. i-cisTarget 2015 update: generalized cis-regulatory enrichment analysis in human, mouse and fly. Nucleic Acids Res 2015; 43: 57-64.

14 Champhekar A, Damle SS, Freedman G, Carotta S, Nutt SL, Rothenberg EV. Regulation of early T-lineage gene expression and developmental progression by the progenitor cell transcription factor PU.1. Genes Dev 2015; 29: 832-848.

15 Koch U, Radtke F. Notch in T-ALL: New players in a complex disease. Trends Immunol 2011; 32: 434-442.

16 Zuurbier L, Petricoin EF, Vuerhard MJ, Calvert V, Kooi C, Buijs-Gladdines JGCAM et al. The significance of PTEN and AKT aberrations in pediatric T-cell acute lymphoblastic leukemia. Haematologica 2012; 97: 1405-1413.

17 Janssen JW, Ludwig WD, Sterry W, Bartram CR. SIL-TAL1 deletion in T-cell acute lymphoblastic leukemia. Leukemia 1993; 7: 1204-1210.

18 Mansour MR, Abraham BJ, Anders L, Berezovskaya A, Gutierrez A, Durbin AD et al. Oncogene regulation. An oncogenic super-enhancer formed through somatic mutation of a noncoding intergenic element. Science 2014; 346: 1373-1377.

19 Vicente C, Schwab C, Broux M, Geerdens E, Degryse S, Demeyer S et al. Targeted sequencing identifies association between IL7R-JAK mutations and epigenetic modulators in T-cell acute lymphoblastic leukemia. Haematologica 2015; 100: 1301-1310.

20 Mendes RD, Sarmento LM, Canté-Barrett K, Zuurbier L, Buijs-Gladdines JGCAM, Póvoa $\checkmark$ et al. PTEN microdeletions in T-cell acute lymphoblastic leukemia are caused by illegitimate RAG-mediated recombination events. Blood 2014; 124: 567-578.

21 Liu Y, Easton J, Shao Y, Maciaszek J, Wang Z, Wilkinson MR et al. The genomic landscape of pediatric and young adult T-lineage acute lymphoblastic leukemia. Nat Genet 2017; 49: 1211-1218.

22 Clappier E, Gerby B, Sigaux F, Delord M, Touzri F, Hernandez L et al. Clonal selection in xenografted human $T$ cell acute lymphoblastic leukemia recapitulates gain of malignancy at relapse. J Exp Med 2011; 208: 653-661.
23 Sanda T, Lawton LN, Barrasa MI, Fan ZP, Kohlhammer H, Gutierrez A et al. Core transcriptional regulatory circuit controlled by the TAL1 complex in human T cell acute lymphoblastic leukemia. Cancer Cell 2012; 22: 209-221.

24 Gerby B, Tremblay CS, Tremblay M, Rojas-Sutterlin S, Herblot S, Hébert J et al. SCL, LMO1 and Notch1 Reprogram Thymocytes into Self-Renewing Cells. PLoS Genet 2014; 10: e1004768.

25 Ivanova NB, Dimos JT, Schaniel C, Hackney JA, Moore KA, Lemischka IR. A stem cell molecular signature. Science 2002; 298: 601-604.

26 Gal H, Amariglio N, Trakhtenbrot L, Jacob-Hirsh J, Margalit O, Avigdor A et al. Gene expression profiles of $\mathrm{AML}$ derived stem cells; similarity to hematopoietic stem cells. Leukemia 2006; 20: 2147-2154.

27 Ferrando AA, Neuberg DS, Staunton J, Loh ML, Huard C, Raimondi SC et al. Gene expression signatures define novel oncogenic pathways in T cell acute lymphoblastic leukemia. Cancer Cell 2002; 1: 75-87.

28 Herranz D, Ambesi-Impiombato A, Sudderth J, Sánchez-Martín M, Belver L, Tosello $\mathrm{V}$ et al. Metabolic reprogramming induces resistance to antiNOTCH1 therapies in T cell acute lymphoblastic leukemia. Nat Med 2015; 21 : 1182-1189.

29 Oliveira ML, Akkapeddi P, Alcobia I, Almeida AR, Cardoso BA, Fragoso R et al. From the outside, from within: Biological and therapeutic relevance of signal transduction in T-cell acute lymphoblastic leukemia. Cell Signal 2017; 38: $10-25$.

30 Sharfe N, Dadi HK, Roifman CM. JAK3 protein tyrosine kinase mediates interleukin-7-induced activation of phosphatidylinositol-3' kinase. Blood 1995; 86: 2077-2085.

31 Barata JT, Silva A, Brandao JG, Nadler LM, Cardoso AA, Boussiotis VA. Activation of $\mathrm{PI} 3 \mathrm{~K}$ is indispensable for interleukin 7-mediated viability, proliferation, glucose use, and growth of T cell acute lymphoblastic leukemia cells. J Exp Med 2004; 200: 659-669.

32 Hagenbeek TJ, Naspetti M, Malergue F, Garçon F, Nunès JA, Cleutjens KBJM et al. The loss of PTEN allows TCR a $\beta$ Lineage thymocytes to bypass IL-7 and pre-TCRmediated signaling. J Exp Med 2004; 200: 883-894.

33 Shi J, Petrie HT. Activation kinetics and off-target effects of thymus-initiated Cre transgenes. PLoS One 2012; 7: e46590.

34 Carow B, Gao Y, Coquet J, Reilly M, Rottenberg ME. Ick-driven Cre expression alters $T$ cell development in the thymus and the frequencies and functions of peripheral T cell subsets. J Immunol 2016; 197: 2261-2268.

35 Palomero T, Sulis ML, Cortina M, Real PJ, Barnes K, Ciofani M et al. Mutational loss of PTEN induces resistance to NOTCH1 inhibition in T-cell leukemia. Nat Med 2007; 13: $1203-1210$

36 Kreslavsky T, Gleimer M, Miyazaki M, Choi Y, Gagnon E, Murre C et al. Beta -selection-induced proliferation is required for ab $\mathrm{T}$ cell differentiation. Immunity 2012; 37: 840-853.

37 Peirs S, Matthijssens F, Goossens S, Van Ide W, Ruggero K, de Bock CE et al ABT-199 mediated inhibition of BCL-2 as a novel therapeutic strategy in T-cell acute lymphoblastic leukemia. Blood 2014; 124: 3738-3747.

38 Kotschy A, Szlavik Z, Murray J, Davidson J, Maragno AL, Le Toumelin-Braizat G et al. The MCL1 inhibitor $\mathbf{S 6 3 8 4 5}$ is tolerable and effective in diverse cancer models. Nature 2016; 538: 477-482.

39 Laukkanen S, Grönroos T, Pölönen P, Kuusanmäki H, Mehtonen J, Cloos J et al. In silico and preclinical drug screening identifies dasatinib as a targeted therapy for T-ALL. Blood Cancer J 2017; 7: e604.

40 Martins VC, Busch K, Juraeva D, Blum C, Ludwig C, Rasche V et al. Cell competition is a tumour suppressor mechanism in the thymus. Nature 2014; $\mathbf{5 0 9}$ 465-470.

41 Sanda T, Leong WZ. TAL1 as a master oncogenic transcription factor in T-cell acute lymphoblastic leukemia. Exp Hematol 2017; 53: 7-15.

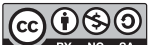

This work is licensed under a Creative Commons AttributionNonCommercial-ShareAlike 4.0 International License. The images or other third party material in this article are included in the article's Creative Commons license, unless indicated otherwise in the credit line; if the material is not included under the Creative Commons license, users will need to obtain permission from the license holder to reproduce the material. To view a copy of this license, visit http:// creativecommons.org/licenses/by-nc-sa/4.0/

(c) The Author(s) 2018

Supplementary Information accompanies this paper on the Leukemia website (http://www.nature.com/leu) 